Document downloaded from:

http://hdl.handle.net/10251/101123

This paper must be cited as:

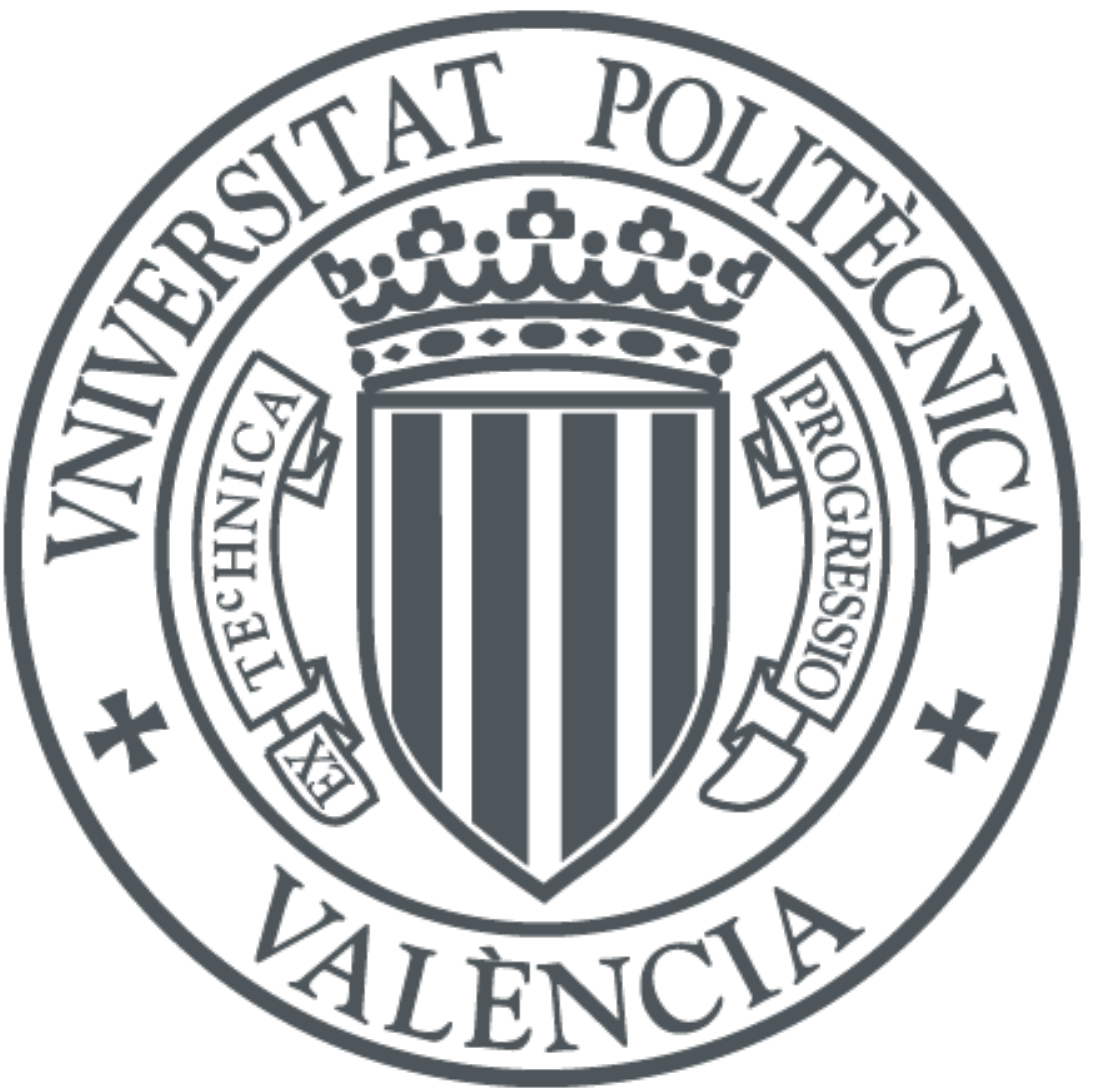

The final publication is available at

https://doi.org/10.1016/j.cemconres.2017.08.011

Copyright Elsevier

Additional Information 


\title{
Ultrasonic Signal Modality: A novel approach for concrete damage evaluation
}

\author{
A. Carrión ${ }^{\mathrm{a}, *}$, V. Genovés ${ }^{\mathrm{b}, * *}$, J. Gosálbez ${ }^{\mathrm{a}}, \mathrm{R}$. Miralles $^{\mathrm{a}}$, J. Payáb $^{\circ}$ \\ ${ }^{a}$ ITEAM, Universitat Politècnica de València, Camino de Vera, $s / n 46022$ Valencia, Spain \\ ${ }^{b}$ ICITECH, Universitat Politècnica de València, Camino de Vera, s/n 46022 Valencia, Spain
}

\section{Abstract}

In this paper, a new approach for characterizing material damage, using ultrasonic waves, is proposed. Two concrete series with two types of cement with different $\mathrm{C}_{3} \mathrm{~A}$ content and similar mechanical properties were subjected to external sulphate attack (ESA) and evaluated using a novel Recurrence Plot Quantification Analysis (RQA) method. This brand new technique was compared with several methods, such as mechanical tests (compressive and flexural strength determination), dynamic test (dynamic modulus) measurements, and traditional ultrasonic measurements (propagation velocity and ultrasonic wave attenuation). In these experiments, RQA showed a high sensitivity to damage in spoiled series, improving the reliability of damage detection with ultrasonics in non-homogeneous materials compared to other non-destructive techniques. Interesting advantages of this new non-destructive technique are: a) the RQA parameter is normalized (range of 0 to 1 ); b) a calibration process is not required; c) the values of its standard deviation show the dispersion of the damage. It can contribute greatly to the diagnosis of the degree of damage to a material, when combined with other traditional measures such as the attenuation of the material.

\section{Graphical abstract}
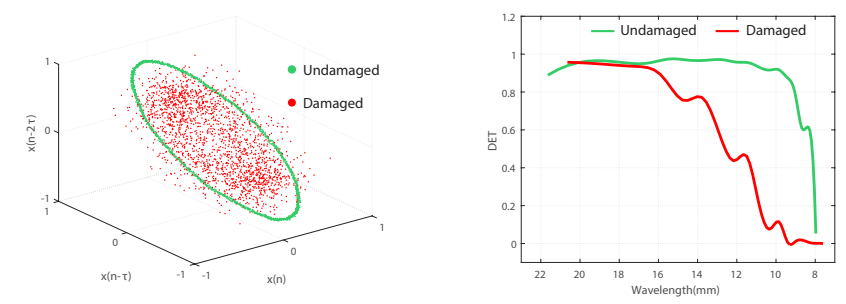

Keywords:

NDT, Recurrence Quantification Analysis, Concrete, Ultrasonics, Damage, Signal Modality, Frequency sweep

\footnotetext{
*Corresponding author E-mail adress: carriongarcia.a@gmail.com

**Corresponding author E-mail adress: genoves.gomez@gmail.com
} 


\section{Introduction}

Concrete is the most widely used synthetic material all over the world in civil and building engineering and the kind of mechanical and physico-chemical properties required by the project. This material has an excellent mechanical and structural performance. However, it has important issues in terms of durability when exposed to different environments and harsh conditions. Freeze-thawing cycles, internal sulphate attack (ISA), external sulphate attack (ESA), overload cracking and wet-drying cycles are some of the processes which require early damage detection in order to avoid serious problems involving the structural elements made with concrete.

Of all the problems previously mentioned, in the present research, ESA was selected to damage the concrete, due to its micro-cracking and expansive process (produced by the formation of secondary ettringite), which globally affects the cement paste structure, weakening the different interfaces, and consequently reducing the physical and mechanical integrity of the material. Initially, this sulphate bearing product fills the voids and capillary network, as well as promoting an increase in the compressive strength [1]. When the voids and free space in the binding matrix are full, micro-cracks start to appear and the compressive strength and Young's modulus of the concrete decrease. When this process is highly aggressive, the cement paste could lose strength and adhesion capacity due to decalcification of C-S-H [2]. Due to the aggressive action of ESA, premature and accurate detection of the spoiling of concrete elements has become an important issue for many research projects.

Damage characterization techniques can be classified into either local or global methods. It is believed that these two methods should be used in a complementary way to effectively and correctly assess the integrity of a complex structure [3]. Most currently used techniques, such as visual, acoustic, magnetic field, electrical etc., are effective yet local in nature $[4,5]$. They mainly require that the vicinity of the damage is known a priori and the portion of the structure being inspected is readily assessible. Methods like Electrical Resistance Tomography have been recently investigated showing high sensitivity to discrete cracks and heterogeneties from different natures like iron bars or discrete cracks performed with plastic plates [6, 7]. On the other hand, global methods quantify the healthiness of a structure (or part of it) by examining changes in its vibrational characteristics or changes in ultrasonic vaves propagation. The standard method for detecting defects within a concrete structure involves the use of drilled core samples [8]. Some of the traditional methods are strength tests, three point bending tests, the determination of the elastic modulus, etc. These methods are the most immediate approaches, but have the disadvantage of being expensive and allowing the analysis of only a small portion of the structure. Hence, there is a need for nondestructive research methods in the field of material characterization and damage detection. Owing to the non-invasive nature, Non Destructive Test (NDT) techniques appear as a reliable tool to detecting and characterise damage of different typology. Recent studies are focused in detecting nonlinearities in mechanical waves in cementitious materials due to the sensitivity to damage either with vibration spectroscopy and ultrasonics. Nonlinear Elastic Wave Spectroscopy techniques are based on the nonlinear decreasing of the 
fundamental frequency with the increasing amplitude of excitation. With this techniques, thermal damage, alkalisilica reaction, sulphate attack and other distributed damages in concrete has been successfully monitored [9-12]. Acoustic Emission techniques also exhibited good results detecting the focus of microcracking process and flaws location when energy is released during mechanical tests [13, 14].

Because of its heterogeneous nature, concrete contains micro-defects even before the occurrence of any damage. Therefore, ultrasonic waves traveling through concrete are both modulated and attenuated as a consequence of the material's inhomogeneity. In addition, scattering occurs at each interface between the aggregate and the matrix [15]. Thus, the travelling waveform exhibits a decrease in amplitude due to the scattering process at each matrix-aggregate interface. The most common ultrasonic measurements are the propagation velocity (ultrasonic pulse velocity, UPV) and the ultrasonic waveform attenuation introduced by the material, both based on the linear theory of the propagation signal (the frequency components of the emitted pulse and the received pulse are similar). Recent studies contributed to exploit the information of UPV with ultrasonic imaging and travel wave tomography to characterise mechanical and freeze-thaw cycles damage [16, 17]. However, the presence of nonlinear terms in the elastic response of a granular medium is considered an indicator related to the presence of any kind of damage. Many techniques have been presented to extract nonlinear features from ultrasounds based on many different approaches, such as higher-order harmonics [18], subharmonics [19], or sidebands [20]. Nonlinear ultrasonics are also being studied by the Scaling Substraction Method, a novel technique that allows to correlate damage with the loss of linearity based on amplitude scaling in a through-transmission layout $[21,22]$. Also, ultrasonic surface wave has been used to detect global micro-cracking processes in concrete elements [23, 24], even with air-coupling layout [25].

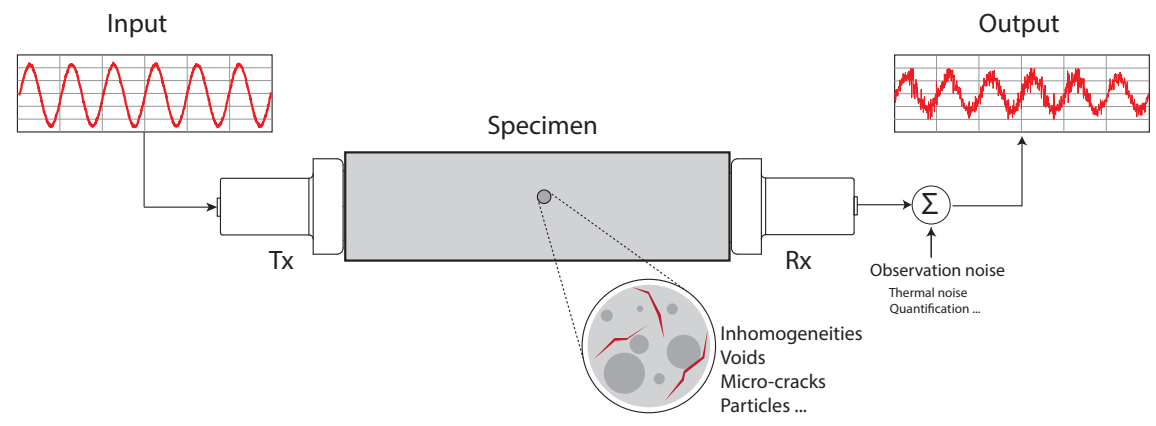

Figure 1: Typical ultrasonic inspection of a specimen and examples of input and output signals. The received signal results from many superimposed echoes scattered by the heterogeneous microstructure of the material.

In the present paper, a new signal modality approach for material characterization is presented. When considering the characterisation of the signal modality, we adhere to the changes in the nature of real world signals: the degree of sparsity, nonlinearity, stochasticity, etc. The applications of signal modality characterisation have only recently become apparent in signal processing and machine learning and are a key topic of multidisciplinary research [26]. In the case at hand, the concept is applied to analyse the interaction between the injected ultrasonic 
wave and the nonlinear scatters placed at the concrete specimen under study. Figure 1 illustrates a typical ultrasonic inspection where the input signal has a predominant deterministic component (coherent component) and the output is the sum of many superimposed echoes scattered by the heterogeneous microstructure of the material (incoherent components). The resulting stochastic nature of the received signal comes not only from the randomly placed reflections, which cause significant changes in amplitude, but also from the echoes transmitted by nonlinear coupling, which combine with different initial phases. Measuring the degree of predictability of the received signals can give information about how the coherent and incoherent components are combined as a function of the inner microstructure and, thus, about the internal damage. The aim of the proposed method is to provide a quantitative measure of the growth of the damage, assessing any change in the material integrity as a whole. The results obtained from some concrete specimens exposed to an ESA attack are analysed and discussed in order to make an initial assessment of the performance of the new proposed measurement method.

The remainder of this paper is organized as follows. The new approach based on the signal modality characterization is discussed in Section 2. Section 3 presents experimental issues, the tested materials as well as the traditional ultrasonic measurements carried out to characterize them. Section 4 describes the results obtained from the application of destructive tests and the ultrasonic measurements to the specimens under testing. The results of the predictability are discussed and compared to the previously known techniques. Finally, the conclusions are summarized in Section 5 .

\section{Recurrence Plot Quantification Analysis (RQA)}

An ultrasonic wave has numerous interactions with the internal structure of the material and the data derived from such observations is related to the behaviour of the material at different structural scales (from the macro- to the micro-level). The concept of multi-scale interactions can be described starting at the level of atomic bonds, its nanometric scale (gel pores, the capillary net mainly made of portlandite and calcium aluminate hydrates), and including that of the mesoscopic (microscopic and millimetric) structure, the aggregate dimension, and finally the concrete structure as a single component [27]. The advantage of ultrasonic waves is that they allow access to every level of these interactions by changing the injected frequency, thereby providing a reliable means of characterising the material at each scale.

The new proposed technique aims to quantify the non-coherent waves produced by echoes from the edges and faces of the specimen, as well as the effects of scattering from the aggregates. Both the echoes and the scattering effects strongly depend on the structural scale, and therefore on the working frequency. In particular, the scattering component of the global attenuation of the ultrasonic wave is well-known to be determined as a function of the input frequency (or its corresponding wavelength, $\lambda$ ) and the average size of the aggregates $(\bar{D})$. Three different regions have been identified: the Rayleigh field $(\lambda \ll \bar{D})$, the stochastic field $(\lambda \simeq \bar{D})$, and the geometric field $(\lambda>\bar{D})$ [28]. The stochastic component that appears depends on the aforementioned ratio and, somehow, summarizes the inhomogeneities seen at the analysed scale and present in the specimen under study. 
Quantifying the degree of predictability of the time series based on the concept of characterization of the modality of the signal [29] can provide information about the signal strength of the input deterministic component in relation to the whole of the acquired time series.

The study of a deterministic signal has relied on the concept of phase space, which is a vector space that collects all the possible system states that are useful for determining the future evolution of the signal. For a time series $x(n)$, the phase space would be defined by

$$
\begin{array}{r}
\vec{X}_{n}=[x(n), x(n-\tau), \ldots, x(n-(E-1) \cdot \tau)]^{T}, \\
n=1, \ldots, N-(E-1) \cdot \tau
\end{array}
$$

where $N$ is the total number of points, $\tau$ is the discrete time lag, $E$ is the embedding dimension [30, 31], and $T$ refers to the transposed matrix. The proper selection of $\tau$ and $E$ is crucial in the further analysis because it affects the correct representation of the data's evolution in time. A common approach to determining the value of $\tau$ is the one proposed by Fraser and Swinney [32], which uses the first null of the time delayed mutual information. The selection of the minimum embedding dimension $E$ is based on the false nearest neighbor algorithm proposed by Cao [33]. In the present paper, the value of the embedding dimension $E$ is chosen as the fraction of false points smaller than $2 \%$. Any other methods [34] for the estimation of $E$ and $\tau$ might be used and the results are not strongly influenced if both parameters are properly computed.

Eckmann et al. [35] introduced a tool called Recurrence Plots (RP) to visualize the recurrence of states which make up the phase space of a signal $x(n)$. The quantification of the number and duration of the recurrences allows us to study the degree of determinism [36] as well as some other characteristics of the underlying dynamic system: laminar phases [37], unstable periodic orbits [38], etc. One of the main advantages of the RP is that they allow the $E$-dimensional phase-space trajectory, $\vec{X}_{n}$, of a signal $x(n)$ to be investigated through a binary two-dimensional representation of the recurrences of the states. Among the different variations of computing the $\mathrm{RP}$, the most common way is using Eq. 2,

$$
R_{n_{1}, n_{2}}=\Theta\left(\varepsilon-\left\|\vec{X}_{n_{1}}-\vec{X}_{n_{2}}\right\|\right), \quad n_{1}, n_{2}=1, \ldots, N_{s}
$$

where $N_{s}$ is the number of considered states $\vec{X}_{n}, \varepsilon$ is a threshold distance, $\|\cdot\|$ is the Euclidean distance, and $\Theta(\cdot)$ is the Heaviside step function.

A diagonal line in the RP appears when a segment of the trajectory runs parallel to another segment. The length of this diagonal line is determined by the number of consecutive states for which two trajectory segments have a similar evolution. As a result, the presence of diagonal lines that run parallel to the mean diagonal (the line of identity) indicates that the evolution of states is similar at different times and that the process could be deterministic. The percentage of recurrence points that form diagonal lines can be used to measure the degree of determinism (DET) [37]. This percentage can be computed as follows:

$$
D E T=\frac{\sum_{j=j_{\min }}^{N_{s}} j \cdot P(j)}{\sum_{j=1}^{N_{s}} j \cdot P(j)}
$$




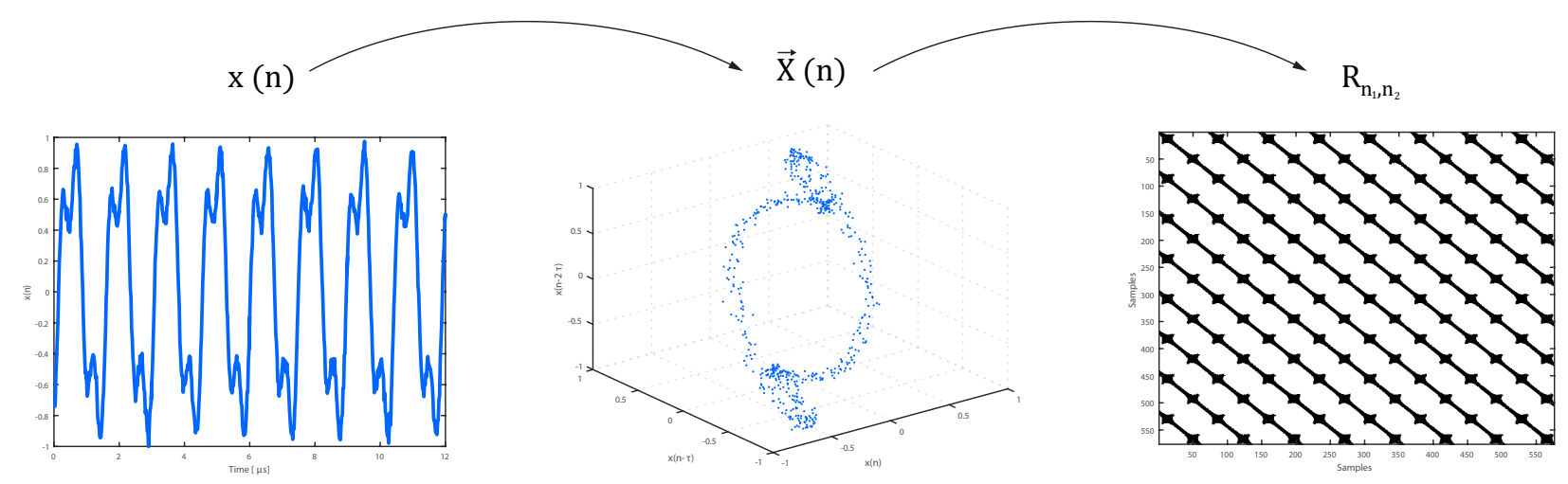

Figure 2: Steps of Recurrence Plot Quantification Analysis (RQA): a) time representation of the signal $x(n)$; b) phase space representation of the signal, $\vec{X}$; c) computation of the recurrence plot $R_{n_{1}, n_{2}}$. In this example, $x(n)$ is obtained from a real ultrasonic inspection when the input is a square waveform. The phase space is obtained using $E=3$ and $\tau=4$.

where $P(j)$ is the number of diagonal lines of length $j$ and $j_{\min }$ is the minimum number of points to be considered as a diagonal line (in the present paper, $j_{\min }=2$ is used).

Figure 2 summarizes the generic steps of the recurrence plots analysis for a signal $x(n)$. First is the time representation of the acquired ultrasonic signal, $x(n)$. Second, the phase space reconstruction, $\vec{X}$, using the optimal parameters $E$ and $\tau$. Third, the computation of the recurrence plot, $R_{n_{1}, n_{2}}$, over which the degree of determinism is computed. A time series $x(n)$ is said to be deterministic if its trajectory in the phase space is smooth and can be modelled as a continuous function. There results a RP matrix where almost every state is recurrent and has long diagonal lines. Therefore, the value of the parameter DET is close to 1 . Otherwise, if the time series is stochastic, the values of DET are close to 0 . The intermediate case corresponds to a signal with both deterministic and stochastic components.

The variation of the degree of determinism related to the frequency of the transmitted ultrasonic wave allows characterising the heterogeneous nature of concrete. DET values close to 1 mean that the received signal has a strong deterministic component linked to the input signal which has been little altered, however, DET values close to 0 mean that the stochastic component predominates. The evolution of the DET along the frequency (or the equivalent wavelength) allows identifying the different structural scales of the material.

\section{Experimental}

Traditional methods for material damage detection were used in this experimental study following the established standards: a three point bending test (Instron universal testing machine, model 3382), compressive strength test (Ibertest MEH LC MD2 W), and transversal dynamic elastic modulus test (according to the ASTM C 21502 standard [39]), for comparison with typical ultrasonic parameters and the new proposed technique based on the Recurrence Plot Quantification Analysis (RQA). 


\subsection{Materials and specimens}

Table 1 shows the chemical composition of both types of cement used in this experiment. The main difference between them is the $\mathrm{C}_{3} \mathrm{~A}$ content: the sulphate resistant cement was a Spanish Portland cement CEM I-52,5 SR (in this paper, denoted by ' $G$ ') and it has $2.33 \% \mathrm{C}_{3} \mathrm{~A}$, and white Portland cement, BL II A-LL 42.5 R, with 9.84\% $\mathrm{C}_{3} \mathrm{~A}$ (in this paper denoted by 'W').

Table 1: Cement composition by \% weight.

\begin{tabular}{llllllllllll}
\hline Cement type & $\mathrm{LOI}^{*}$ & $\mathrm{SiO}_{2}$ & $\mathrm{Al}_{2} \mathrm{O}_{3}$ & $\mathrm{Fe}_{2} \mathrm{O}_{3}$ & $\mathrm{CaO}$ & $\mathrm{MgO}$ & $\mathrm{SO}_{3}$ & $\mathrm{C}_{4} \mathrm{AF}$ & $\mathrm{C}_{3} \mathrm{~A}$ & $\mathrm{C}_{3} \mathrm{~S}$ & $\mathrm{C}_{2} \mathrm{~S}$ \\
\hline CEM I-52,5 SR & 2.05 & 20.52 & 3.37 & 3.92 & 63.36 & 1.96 & 2.59 & 11.93 & 2.33 & 55.7 & 16.94 \\
BL II A-LL 42.5 R & 9.75 & 16.55 & 3.88 & 0.26 & 62.91 & 1.39 & 4.28 & 0.8 & 9.84 & 51.73 & 8.56 \\
\hline
\end{tabular}

* Loss On Ignition

A plain 0.65 water/cement ratio concrete was designed for this experiment varying the cement type in order to obtain two materials with different chemical properties with respect to ESA. The dosage used for each series is shown in Table 2.

Table 2: Concrete dosage in $\mathrm{kg}$.

\begin{tabular}{lllllll}
\hline Series & Cement type & Cement & Water & Gravel 7/12 & Gravel 4/7 & Sand 0/4 \\
\hline GT0 \& GT1 & CEM I-52,5 SR & 5.25 & 3.41 & 5.44 & 5.44 & 16.33 \\
WT0 \& WT1 & BL II A-LL 42.5 R & 5.25 & 3.41 & 5.44 & 5.44 & 16.33 \\
\hline
\end{tabular}

After properly mixing the raw materials, ten $60 \times 60 \times 240 \mathrm{~mm}^{3}$ moulds per each series were filled with fresh concrete and stored in a wet chamber $\left(20^{\circ} \mathrm{C}\right.$ and $\left.100 \% \mathrm{HR}\right)$ for 28 days. After that and before the ESA, five samples of each cement type were used to do mechanical test (GT0 and WT0). The remaining five samples of each series were tested by ultrasounds (GT0 and WT0) before storing them in the wet chamber immersed in tanks with a $10 \% \mathrm{Na}_{2} \mathrm{SO}_{4}$ solution for 28 days. Afterwards, the samples were ultrasonic characterized (GT1 and WT1), and finally, broken in the later mechanical tests (GT1 and WT1). Therefore, the same five different samples were characterized by ultrasounds before and after ESA.

\subsection{Ultrasonic measurements}

An ultrasonic through-transmission setup was chosen since it offers good penetration and accuracy for the ultrasound measures estimated from the received signal: the p-wave velocity and the attenuation due to the material [40-42]. For non-continous waves of a specific frequency, $f$, the propagation velocity (or wavefront speed), $v_{p}(f)[\mathrm{m} / \mathrm{s}]$, is defined as the speed with which the beginning of the disturbance moves. This value equals the phase velocity and the group velocity as long as they are independent of the wavelength (non-dispersive materials). The computed $v_{p}(f)$ is given by Eq. 4 ,

$$
v_{p}(f)=\frac{d_{m a t}}{t_{a}(f)}
$$


where $t_{a}(f)[s]$ is the time that the emitted wave takes to pass through the material (whose distance is $d_{\text {mat }}[\mathrm{m}]$ ) and it is estimated as the instant of time when the received signal level exceeds $50 \%$ of the noise level. This time is proportional to the mechanical constants of the material, and therefore, to the movement of the particles forming the material caused by the applied external forces. The calibration procedure related to the velocity estimation implies a time of flight correction for the K0.5SM transducers (General Electric) equals to $1.64 \mu \mathrm{s}$.

The examination of the amplitude of the signals at different frequencies revealed interesting features related to the aggregate content and size. The attenuative behaviour of the material implies both an intrinsic (absorption) and an extrinsic (scattering) mechanism, which cannot be directly separated. The attenuation coefficient, $\alpha_{\text {mat }}(f)[\mathrm{dB} / \mathrm{cm}]$, is determined by measuring the reduction of the amplitude of a sinusoidal permanent wave and is given by Eq. 5 ,

$$
\begin{aligned}
\alpha_{\text {mat }}(f)[\mathrm{dB} / \mathrm{cm}] & =\frac{P_{t x}(f)[\mathrm{dB}]-P_{r x}(f)[\mathrm{dB}]-\alpha_{\text {equip }}(f)[\mathrm{dB}]}{d_{\text {mat }}[\mathrm{cm}]} \\
P_{t x}[\mathrm{~dB}] & =10 \cdot \log _{10}\left(\frac{A_{t x}^{2}}{2}\right) \\
P_{r x}[\mathrm{~dB}] & =10 \cdot \log _{10}\left(\frac{A_{r x}^{2}}{2}\right)=10 \cdot \log _{10}\left(\frac{\max \left\{s_{r x}(t)\right\}^{2}}{2}\right)
\end{aligned}
$$

where $P_{t x}[\mathrm{~dB}]$ is the transmitted power and can be obtained theoretically from the amplitude of the transmitted signal, $A_{t x}$, using Eq. (6), $P_{r x}(f)[\mathrm{dB}]$ is the received power and is obtained from Eq. (7) where $s_{r x}(t)$ is the received signal, $\alpha_{\text {equip }}[\mathrm{dB}]$ is the attenuation of the equipment, and $d_{\text {mat }}[\mathrm{cm}]$ is the total length of the specimen.

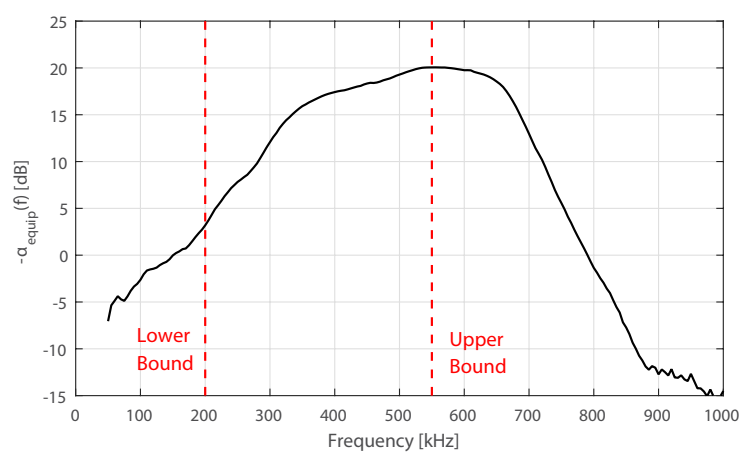

Figure 3: Frequency response of the measuring equipment $\left(-\alpha_{\text {equip }}(f)\right)$. Red lines represent the lower (200 kHz) and upper (550 kHz) bounds of the working interval.

The attenuation associated to the measurement equipment (transducers, amplifier, wires, acquisition module,...) requires a calibration process. The calibration process was carried out by having emitter and receiver transducers facing each other without any material between them and applying the simplified version of Eq. 5, 
namely, Eq. 8. Figure 3 plots the resulting frequency response of the used equipment, $-\alpha_{\text {equip }}(f)$.

$$
\alpha_{\text {equip }}(f)[\mathrm{dB}]=P_{t x}(f)[\mathrm{dB}]-P_{r x}(f)[\mathrm{dB}]
$$

The transducers employed for transmission and reception were the K05SM from General Electric, broadband transducers whose bandwidth is centred at $500 \mathrm{kHz}$. Pure vaseline was used as coupling agent. The transmitter transducer was excited directly by a programmable signal generator (Agilent 33120A). The received and amplified ultrasonic signal was captured by a digital oscilloscope (Tektronix DPO3014) with a sampling frequency of 50 MHz. And finally, a laptop was used to control the signal generator and to acquire and store the digitized signals by the oscilloscope (see Figure 4 for the schematic layout of the experiment).

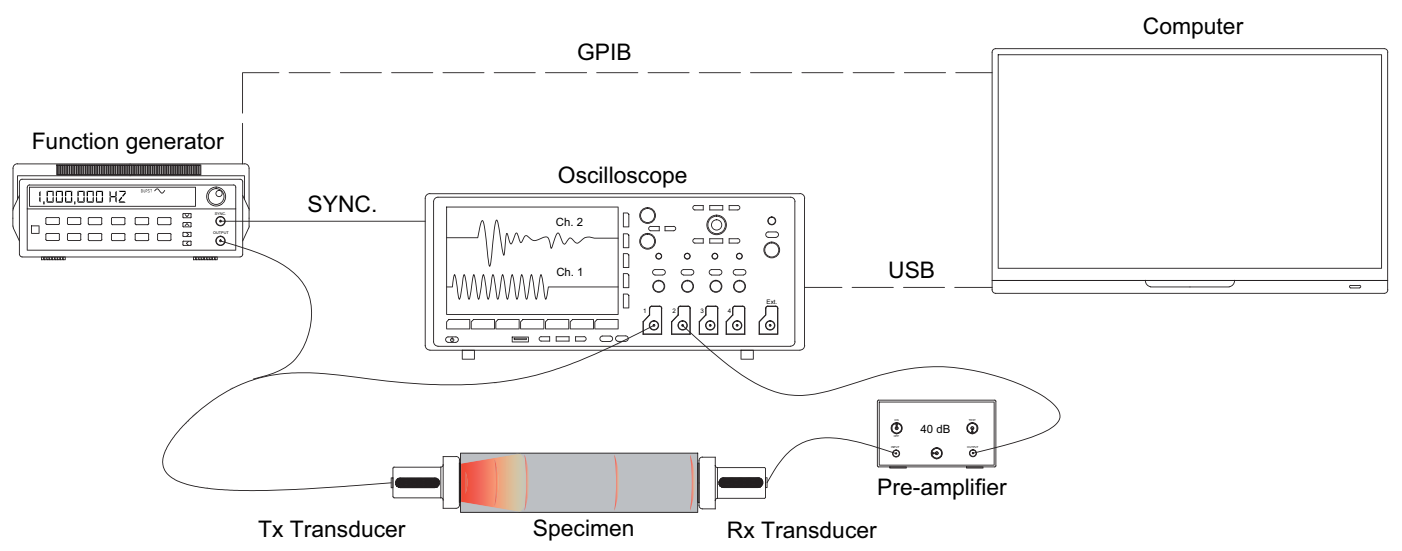

Figure 4: Ultrasonic equipment layout: transmission and reception transducers, programmable signal generator, pre-amplifier, digital oscilloscope and a laptop.

The transmitted signals were different in each performed analysis: a 5-cycle sinusoidal tone burst signal for time of flight estimation and a sinusoidal signal for attenuation and signal modality measurements. The wave parameters in both signals were: amplitude, $\mathrm{A}_{t x}=10 \mathrm{~V}$, and the fundamental frequency, $f_{0}$, was swept from 200 $\mathrm{kHz}$ to $550 \mathrm{kHz}$ in $5 \mathrm{kHz}$ steps. Two aspects were considered for determining the lower bound $(200 \mathrm{kHz})$ of the frequency range: the surface waves generated and the frequency response of the transducers. For the first one, the maximum ultrasound wavelength $\lambda$ was chosen to be smaller than the cross section of the specimens $(60 \mathrm{~mm})$, so that the wave did not propagate across its entire volume. Otherwise, if the entire volume of the specimen is affected, Lamb waves are generated and the propagation velocity becomes dependent on the input frequency. Secondly, the frequency response of the equipment, and primarily that of the transducers, limits the working bandwidth. Regarding Figure 3, the transducers experienced an efficiency loss and the measurements became unstable under a $200 \mathrm{kHz}$ threshold. On the other hand, the upper bound $(550 \mathrm{kHz})$ of the frequency range was chosen considering the amplitude level of the received signal for the less dispersive series (GT1). For this series (GT1), frequencies exceeding $550 \mathrm{kHz}$ were highly attenuated. Therefore, the time of flight as well as the attenuation were estimated inaccurately due to the low signal to noise ratio. 


\section{Results and discussion}

The characterization methods, mechanical properties and traditional ultrasonic measurements are well known methods, and they are used in this section to analyze the results of the new technique.

\subsection{Mechanical and physical properties}

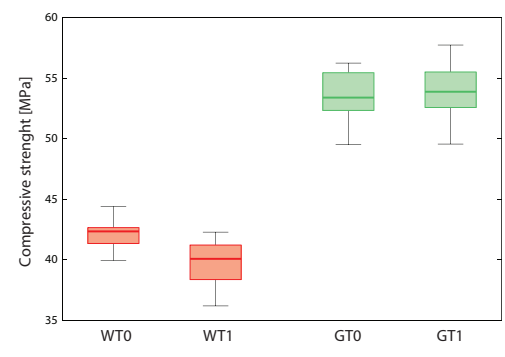

(a)

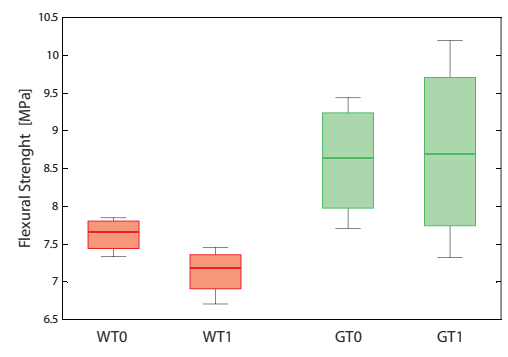

(b)

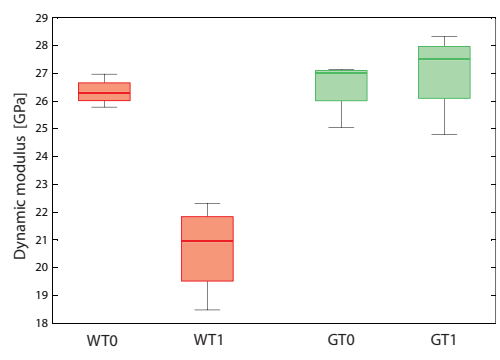

(c)

Figure 5: Mechanical results obtained for each series. (a) Compressive strength test. (b) Three-point bending test. (c) Transversal dynamic elastic modulus test.

\subsection{Ultrasonic results}

\subsubsection{Traditional ultrasonic measurements}

Typical parameters extracted from the ultrasonic wave in time and frequency domains are represented in Figure 6. Figure 6a shows the propagation velocity through the different concrete series. The chosen frequency 


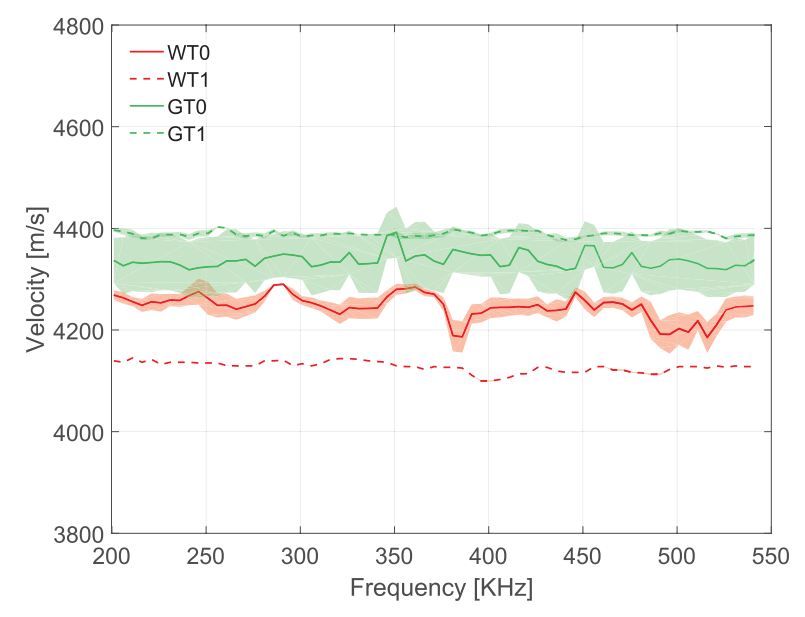

(a)

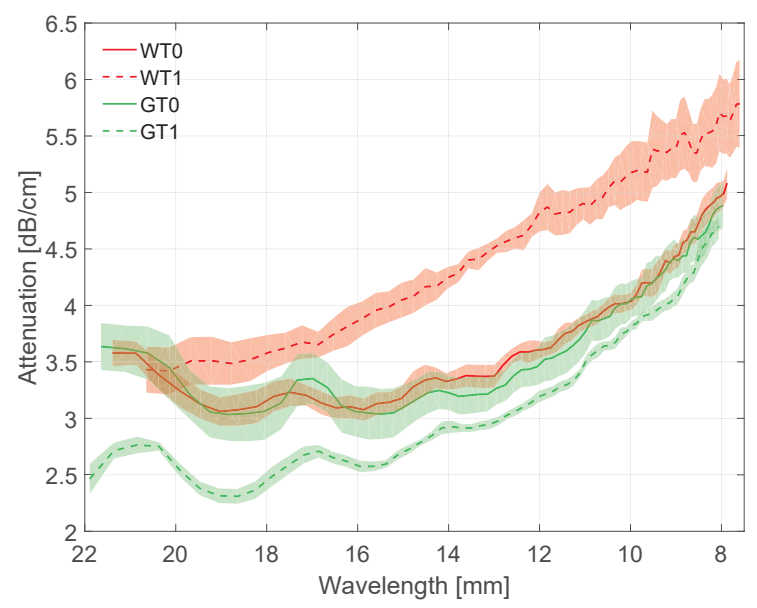

(b)

Figure 6: Mean values (lines) and standard deviations (shaded areas) of typical ultrasonic parameters extracted from signals. The $x$-axis represents the frequency sweep of the input signal expressed in terms of the frequency [kHz], or its corresponding wavelength [mm] stepped by $5 \mathrm{kHz}$. (a) Ultrasonic p-wave velocity. (b) Attenuation of the material.

initial state, GT0 had a greater propagation velocity than WT0 due to the type of cement used in each mix. However, their behaviour after the ESA was completely different, since the G series had better durability than the W ones. WT1 shows lower values of velocity than WT0 because ESA damaged its internal structure significantly, as was previously analysed by means of the mechanical results. Nevertheless, GT1 had an increase in its wavefront velocity due to the effect of the initial stage of ESA. The ettringite formed in the pores and capillary network filled the voids and reinforced the microstructure of the material, making it more compact. This behaviour could be supported by the results shown in Figure $5 c$, that suggest a slight improvement in the stiffness of the material.

Although concrete has been reported previously to exhibit dispersive behaviour in through-transmission layouts, the results of the present paper reveal that the velocity can be considered a constant (differing by about $1 \%)$ at enough working frequencies that are high compared to the size of the inhomogeneities. Notice that the standard deviations for the WT1 and GT1 series are so low that the shaded areas are almost indistinguishable. Hereafter, the characteristic velocities (the average value) for each series are taken: $v_{p, W T 0}=4250 \mathrm{~m} / \mathrm{s}, v_{p, W T 1}$ $=4128 \mathrm{~m} / \mathrm{s}, v_{p, G T 0}=4338 \mathrm{~m} / \mathrm{s}$ and $v_{p, G T 1}=4389 \mathrm{~m} / \mathrm{s}$.

The attenuation strongly depends on the reflectivity of the material, which is related to the scattering (inhomogeneities) due to the microstructure. This relation makes the attenuation a parameter that depends on the size of the reflectors relative to the wavelength of the input signal. The wavelength $(\lambda)$ and frequency $(f)$ of a signal are related through the expression $\lambda=v / f$, where $v$ is the characteristic propagation velocity. Figure 6b shows the attenuation of each series versus the wavelength, ranging from low to high frequencies. The corresponding wavelength has been computed using the characteristic propagation velocity of each series, and it is related to the size of scatterers (reflectors, cracks...) of the inserted material. At higher frequencies (shorter wavelengths), ultrasonic waves suffer a greater number of interactions with the inner structure of the material which, therefore, 
implies higher attenuation.

The initial attenuation for both types of cement, WT0 and GT0, is virtually the same, suggesting the integrity of the matrix of the concrete. However, after ESA, each material follows a different trend. The WT1 series presents a progressively greater attenuation than WT0 as the input frequency increases (wavelength decreases). For instance, for $12 \mathrm{~mm}$ wavelength, WT0 presented $3.5 \mathrm{~dB} / \mathrm{cm}$, whereas WT1 presented $4.8 \mathrm{~dB} / \mathrm{cm}$. This behaviour is attributed to the appearing micro-cracking of the cementing matrix, which becomes more significant in the case of small structures. For the GT1 series, the attenuation decreases after the sulphate attack, a result which supports the previously described filling effect of the capillary network by the formation of sulphate bearing compounds. Since the larger pores have a higher ratio of weight to volume and, therefore, a greater presence in the system, the attenuation significantly decreases at low frequencies (GT0 c. a. $3.5 \mathrm{~dB} / \mathrm{cm}, \mathrm{GT} 1$ c. a. $2.7 \mathrm{~dB} / \mathrm{cm}$ ).

\subsection{2. $R Q A$ results}

As mentioned in Section 2, the degree of determinism must be proportional to the damage suffered by the material. Figure 7 compares the reconstructed phase spaces of four ultrasonic signals at the same excitation frequency, $f_{0}=301 \mathrm{kHz}$, from different cement concrete series. It can be noticed that the trajectories follow similar paths, but each plot has its corresponding stochastic component represented as the dispersion of its points in the phase space. Both cement series, GT0 and WT0, have a similar initial stage (Figures 7a and 7c) but, due to the effect of ESA, each series evolved differently, with the final stage depending on the effect of the formation of expansive products. Figure 7b shows how the series WT1 has a predominant stochastic component, however, Figure $7 \mathrm{~d}$ shows an even stronger deterministic path.

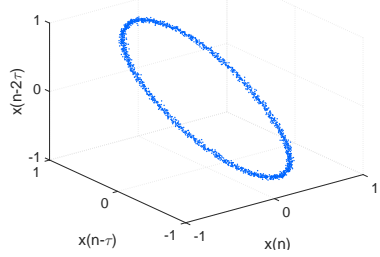

(a)

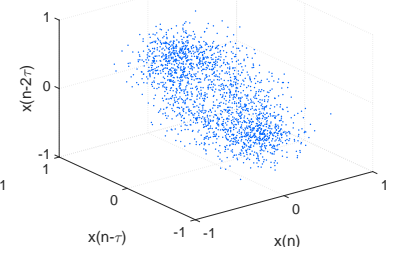

(b)

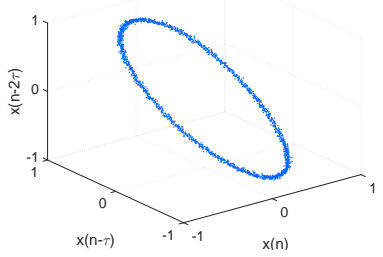

(c)

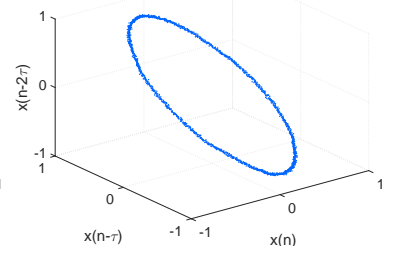

(d)

Figure 7: Phase space representation of four different signals acquired from four different specimens at the same frequency, $f=301 \mathrm{kHz}$. Each phase space is reconstructed using its optimal embedding parameters, $E$ and $\tau$. (a) WT0, (b) WT1, (c) GT0 and (d) GT1.

Accordingly, we must be able to characterize each material by applying the RQA parameter. Figure 8 plots DET as a function of the signal wavelength. For each acquisition, the phase space has been reconstructed using its optimal embedding parameters. Figure 8a represents DET for the W series. Initially, the DET for the WT0 decayed for input signals with wavelengths lower than $10 \mathrm{~mm}$. However after ESA, DET suffers a sudden fall from the $16 \mathrm{~mm}$ wavelength. This result indicates a significant change in the inner structure of the material, to which this parameter is sensitive, from $16 \mathrm{~mm}$. The wide spread of the standard deviation of the DET obtained in the range from $10 \mathrm{~mm}$ to $16 \mathrm{~mm}$ are probably due to the heterogenous cracking process triggered by ESA in 
each specimen of the series. Figure 8b presents DET for the G series, both for the initial (GT0) and final (GT1) states of the experiment. Although the trends remain the same, the latter series has higher DET at every point.

For the GT0, GT1 and WT0 series, the DET curves sharply decrease for higher frequencies (shorter wavelengths) due to the characteristic inhomogeneities of the matrix-aggregate interface. Knowing the aggregates used in concrete dosage (12/7 and 7/4) it could be stablished a wavelength frontier where the determinism drops to 0 values due to the scattering produced by coarse aggregate. If the determinism shows this kind of dispersion, change in the slope or a decrease at higher wavelengths (figure 8a) may be due to the presence of larger heterogeneities induced by a damaging process. Note that the ultrasonic sinusoidal signals used in the RQA analysis have not been compensated in terms of calibration. Nevertheless, the curves maintain high values of DET, and the obtained trends cannot be attributed to the system frequency response seen in Figure 3. Therefore the technique seems to be robust against the equipment involved in the measurement process.

The results obtained by the new proposed method based on RQA have been found to be equivalent to the previously mentioned methods, both mechanical and traditional ultrasonic methods. The new measuring method could be used not only to characterize the damage occurring in materials but also to supplement the information obtained by the attenuation curves. It has been observed that the attenuation can vary both when the frequency increases and if any heterogeneity is present. In the presence of damage (or highly heterogeneous material), the injected signal not only must suffer a significant decrease in amplitude but also random echoes are added to the travelling signal. Therefore, the rise of the attenuation levels must be accompanied by low values of DET.

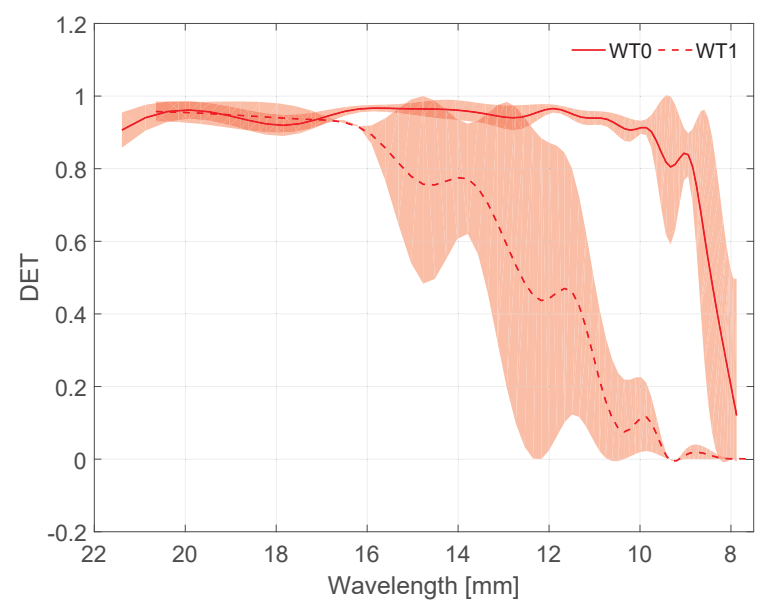

(a)

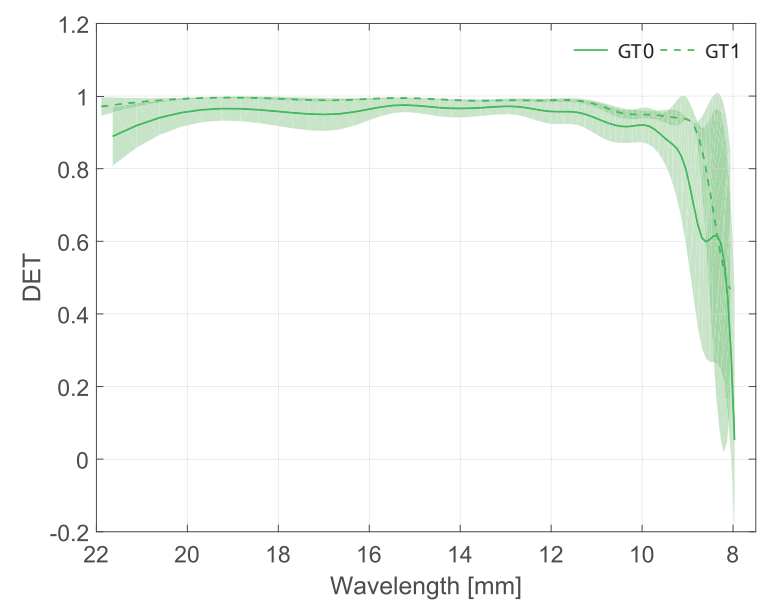

(b)

Figure 8: Mean values (lines) and standard deviations (shaded areas) of the RQA parameter DET extracted from the acquired ultrasonic signals. The $x$ axis represents the frequency sweep of the input signal expressed in terms of the corresponding wavelength [mm], stepped by $5 \mathrm{kHz}$. The phase spaces are reconstructed using their optimal embedding parameters, $E$ and $\tau$. (a) W series. (b) G series. 


\section{Conclusions}

Two concrete series with two types of cement classes with different $\mathrm{C}_{3} \mathrm{~A}$ contents and similar mechanical properties were assessed in terms of External Sulphate Attack (ESA). Several methods were used to correlate mechanical parameters of damage (compressive and flexural strength), dynamic behaviour (dynamic modulus), traditional ultrasonic measurements (p-wave velocity and attenuation) and a brand new method that evaluates the signal modality of ultrasonic waves. Each series developed a different behaviour with the sulphate attack. Concrete made with sulphate resistant cement (G series) kept its mechanical properties, even showing an improvement due to the densification of the microstructure due to the sulphate compounds filling the capillary network and voids. Concrete made with white cement (W series), more vulnerable to ESA due to the higher $\mathrm{C}_{3} \mathrm{~A}$ content, decreased its mechanical performance due to a micro-cracking process promoted by the expansion processes. Mechanical and dynamic tests supported the aforementioned behaviour for all series, decreasing in the case of the $\mathrm{W}$ series and keeping constant or even rising in the case of the G series after the sulphate attack. The p-wave velocity increased in the case of the G series and decreased for the $\mathrm{W}$ series and the attenuation showed the inverse trend, providing additional information for the wavelength range. Recurrence Quantification Analysis (RQA) gave valuable information about the degradation process. In the case of the $\mathrm{W}$ series, non-deterministic values are reached at higher wavelengths than for the concrete before the sulphate attack. Moreover, the damaged W series showed more dispersion (higher standard deviation) in the results than the $\mathrm{W}$ series in the initial stage. The G series remained constant with slight changes in the dispersion of the results. The RQA method showed a significant improvement in damage evaluation techniques (destructive and non-destructive ones) for different reasons: the measure of determinims, DET, is normalized ( 0 to 1 range), a calibration process is not required, its standard deviation shows the damage dispersion, and contributes greatly to diagnose the degree of damage of a material when it is plotted versus the wavelength of the emitted signal. 


\section{Acknowledgements}

This work was supported by the Spanish Government under grants TEC2011-23403, BIA2014-55311-C2-1-P reference P201630212.

\section{References}

[1] C. Ouyang, A damage model for sulfate attack of cement mortars, Cement, Concrete, and Aggregates, CCAGDP, 11(2): 92-99. 14 (1989) 10.

[2] J. Marchand, I. Odler, J. Skalny, Sulfate Attack on Concrete, Modern Concrete Technology, Taylor \& Francis, ISBN 9780203301623, 2004.

[3] Z. Sun, C. Chang, Structural Damage Assessment Based on Wavelet Packet Transform, Journal of Structural Engineering 128 (10) (2002) $1354-1361$.

[4] V. Malhotra, N. Carino, Handbook on Nondestructive Testing of Concrete, Civil engineering, CRC Press, ISBN 9780803120990, 2004.

[5] F. Ghasemzadeh, R. Rashetnia, D. Smyl, M. Pour-Ghaz, A comparison of methods to evaluate mass transport in damaged mortar, Cement and Concrete Composites 70 (2016) $119-129$.

[6] K. Karhunen, A. Seppänen, A. Lehikoinen, P. J. Monteiro, J. P. Kaipio, Electrical Resistance Tomography imaging of concrete, Cement and Concrete Research 40 (1) (2010) 137 - 145.

[7] D. Smyl, R. Rashetnia, A. Seppänen, M. Pour-Ghaz, Can Electrical Resistance Tomography be used for imaging unsaturated moisture flow in cement-based materials with discrete cracks?, Cement and Concrete Research 91 (2017) 61 - 72.

[8] P. Daponte, F. Maceri, R. Olivito, Ultrasonic signal-processing techniques for the measurement of damage growth in structural materials, Instrumentation and Measurement, IEEE Transactions on 44 (6) (1995) 1003-1008.

[9] K. J. Lesnicki, J.-Y. Kim, K. E. Kurtis, L. J. Jacobs, Characterization of ASR damage in concrete using nonlinear impact resonance acoustic spectroscopy technique, NDT \& E International 44 (8) (2011) 721 - 727.

[10] S.-J. Park, H. J. Yim, H.-G. Kwak, Nonlinear resonance vibration method to estimate the damage level on heat-exposed concrete, Fire Safety Journal 69 (2014) $36-42$.

[11] J. Chen, A. R. Jayapalan, J.-Y. Kim, K. E. Kurtis, L. J. Jacobs, Rapid evaluation of alkali-silica reactivity of aggregates using a nonlinear resonance spectroscopy technique, Cement and Concrete Research 40 (6) (2010) 914 - 923.

[12] V. Genovés, L. Soriano, M. Borrachero, J. Eiras, J. Payá, Preliminary study on short-term sulphate attack evaluation by non-linear impact resonance acoustic spectroscopy technique, Construction and Building Materials 78 (2015) 295 - 302.

[13] E. Tsangouri, G. Karaiskos, A. Deraemaeker, D. V. Hemelrijck, D. Aggelis, Assessment of Acoustic Emission localization accuracy on damaged and healed concrete, Construction and Building Materials 129 (2016) 163 - 171.

[14] J. Geng, Q. Sun, Y. Zhang, L. Cao, W. Zhang, Studying the dynamic damage failure of concrete based on acoustic emission, Construction and Building Materials 149 (2017) $9-16$.

R. B. Thompson, Quantitative ultrasonic nondestructive evaluation methods, Journal of Applied Mechanics 50 (4b) (1983) 1191.

[16] M. Molero, S. Aparicio, G. Al-Assadi, M. Casati, M. Hernández, J. Anaya, Evaluation of freeze-thaw damage in concrete by ultrasonic imaging, NDT \& E International 52 (2012) 86 - 94.

[17] Integrated non-destructive assessment of concrete structures under flexure by acoustic emission and travel time tomography, Construction and Building Materials 67 (2014) 202 - 215, 1. Special Issue of KIFA-6 2. Utilization of Crumb Rubber in Asphalt Mixtures.

[18] K. Van Den Abeele, J. De Visscher, Damage assessment in reinforced concrete using spectral and temporal nonlinear vibration techniques, Cement and Concrete Research 30 (9) (2000) 1453-1464.

[19] A. Moussatov, V. Gusev, B. Castagnede, Self-induced hysteresis for nonlinear acoustic waves in cracked material, Physical review letters 90 (12) (2003) 124301.

[20] P. Duffour, M. Morbidini, P. Cawley, A study of the vibro-acoustic modulation technique for the detection of cracks in metals, The Journal of the Acoustical Society of America 119 (3) (2006) 1463-1475.

[21] C. Bruno, A. Gliozzi, M. Scalerandi, P. Antonaci, Analysis of elastic nonlinearity using the scaling subtraction method, Physical Review B 79 (6) (2009) 064108.

[22] P. Antonaci, C. Bruno, P. Bocca, M. Scalerandi, A. Gliozzi, Nonlinear ultrasonic evaluation of load effects on discontinuities in concrete, Cement and Concrete Research 40 (2) (2010) 340 - 346.

[23] J. Li, Y. Lu, R. Guan, W. Qu, Guided waves for debonding identification in CFRP-reinforced concrete beams, Construction and Building Materials 131 (2017) $388-399$.

[24] Q. A. Vu, V. Garnier, J. F. Chaix, C. Payan, M. Lott, J. N. Eiras, Concrete cover characterisation using dynamic acousto-elastic testing and Rayleigh waves, Construction and Building Materials 114 (2016) 87 - 97.

[25] S. Ham, H. Song, M. L. Oelze, J. S. Popovics, A contactless ultrasonic surface wave approach to characterize distributed cracking damage in concrete, Ultrasonics 75 (2017) $46-57$.

[26] B. Jelfs, S. Javidi, P. Vayanos, D. Mandic, Characterisation of Signal Modality: Exploiting Signal Nonlinearity in Machine Learning and Signal Processing, Journal of Signal Processing Systems 61 (1) (2010) 105-115.

[27] V. Garnier, B. Piwakowski, O. Abraham, G. Villain, C. Payan, J. F. Chaix, Acoustic techniques for concrete evaluation: Improvements, comparisons and consistency, Construction and Building Materials 43 (2013) 598 - 613.

[28] W. P. Mason, H. McSkimin, Attenuation and scattering of high frequency sound waves in metals and glasses, The Journal of the Acoustical Society of America 19 (3) (1947) 464-473. 
[29] A. Carrión, R. Miralles, G. Lara, Measuring predictability in ultrasonic signals: An application to scattering material characterization, Ultrasonics 54 (7) (2014) $1904-1911$.

[30] N. H. Packard, J. Crutchfield, J. D. Farmer, R. S. Shaw, Geometry from a Time Series, Phys. Rev. Lett. 45 (9) (1980) 52-56.

$400 \quad$ [31] D. A. Rand, L. S. Young, Dynamical Systems and Turbulence, Springer, Berlin, 1981.

[32] A. Fraser, H. Swinney, Independent coordinates for strange attractors from mutual information, Phys. Rev. A 33 (1986) 1134-1140.

[33] L. Cao, Practical method for determining the minimum embedding dimension of a scalar time series, Physica D: Nonlinear Phenomena $110(1$ - 2) (1997) $43-50$.

[34] T. Gautama, D. P. Mandic, M. M. Van Hulle, A differential entropy based method for determining the optimal embedding parameters of a signal 6 (2003) VI-29.

[35] J.-P. Eckmann, S. O. Kamphorst, D. Ruelle, Recurrence Plots of Dynamical Systems, Europhysics Letters (EPL) 4 (9) (1987) $973-977$.

[36] L. Trulla, A. Giuliani, J. Zbilut, C. Webber Jr., Recurrence quantification analysis of the logistic equation with transients, Physics Letters A 223 (4) (1996) $255-260$.

[37] N. Marwan, N. Wessel, U. Meyerfeldt, A. Schirdewan, J. Kurths, Recurrence-plot-based measures of complexity and their application to heart-rate-variability data, Phys. Rev. E 66 (2002) 026702.

[38] D. P. Lathrop, E. J. Kostelich, Characterization of an experimental strange attractor by periodic orbits, Physical Review A 40 (7).

[39] ASTM, ASTM C1018-97. Standard Test Method for Flexural Toughness and First-Crack Strength of Fiber-Reinforced Concrete ( Using Beam With Third-Point Loading ) 1, ASTM 04 (C) (2011) 1-8.

[40] J. Krautkrämer, H. Krautkrämer, Ultrasonic testing of materials, Springer-Verlag, ISBN 9783540117339, 1983.

415 [41] T. P. Philippidis, D. G. Aggelis, Experimental study of wave dispersion and attenuation in concrete., Ultrasonics 43 (7) (2005) 584-95.

[42] M. Molero, I. Segura, S. Aparicio, M. G. Hernández, M. a. G. Izquierdo, On the measurement of frequency-dependent ultrasonic attenuation in strongly heterogeneous materials., Ultrasonics 50 (8) (2010) 824-8.

[43] I. E. Shkolnik, Evaluation of Dynamic Strength of Concrete from Results of Static Tests, Journal of Engineering Mechanics 122 (December) (1996) 1133-1138.

[44] X. Lu, Q. Sun, W. Feng, J. Tian, Evaluation of dynamic modulus of elasticity of concrete using impact-echo method, Construction and Building Materials 47 (0) (2013) $231-239$. 